\title{
Crystal-chirality-dependent control of magnetic domains in a time-reversal-broken antiferromagnet
}

\author{
Kenta Kimura $\mathbb{D}^{1 凶}$, Yasuyuki Kato $\mathbb{D}^{2}$, Shojiro Kimura ${ }^{3}$, Yukitoshi Motome $\mathbb{D}^{2}$ and Tsuyoshi Kimura (iD)
}

Chiral-lattice magnets can exhibit a variety of physical phenomena when time-reversal symmetry is broken by their magnetism. For example, nonreciprocal responses of (quasi)particles have been widely observed in chiral-lattice magnets with macroscopic magnetization. Meanwhile, time-reversal symmetry can also be broken in antiferromagnets without magnetization. Here we report an unconventional chirality-magnetism coupling in a chiral-lattice antiferromagnet $\mathrm{Pb}(\mathrm{TiO}) \mathrm{Cu}_{4}\left(\mathrm{PO}_{4}\right)_{4}$ whose time-reversal symmetry is broken by an ordering of magnetic quadrupoles. Our experiments demonstrate that a sign of magnetic quadrupoles is controllable by a magnetic field only, which is generally impossible in consideration of the symmetry of magnetic quadrupoles. Furthermore, we find that the sign of magnetic quadrupoles stabilized by applying a magnetic field is reversed by a switching of the chirality. Our theoretical calculations and phenomenological approach reveal that this unusual coupling between the chirality and magnetic quadrupoles is mediated by the previously-unrecognized magnetic octupoles that emerge due to the chirality.

npj Quantum Materials (2021)6:54; https://doi.org/10.1038/s41535-021-00355-0

\section{INTRODUCTION}

Chirality is a common property in nature, describing an asymmetry of an object upon its mirroring. In condensed matter physics, it has been widely known that mirror symmetry breaking in materials with a chiral crystal structure leads to functional properties such as natural optical activity and piezoelectric effects. When time-reversal symmetry is further broken by magnetism, unconventional physical phenomena can emerge. One of the most prominent examples is nonreciprocal propagation and dichroism of elementary particles or excitations, such as photons $^{1,2}$, electrons $s^{3,4}$, magnons ${ }^{5}$, and phonons $s^{6}$. In this case, time-reversal symmetry is broken either by spontaneous macroscopic magnetization (i.e., ferromagnetic order) or by an external magnetic field.

Meanwhile, time-reversal symmetry breaking can occur even in antiferromagnets without macroscopic magnetization. Recent theoretical studies ${ }^{7,8}$ have shown that such time-reversal-broken antiferromagnetic states are fully described by an ordering of particular magnetic multipoles and magnetic toroidal multipoles in terms of their symmetry details. Among them, magnetic quadrupole, which is a second-rank magnetic multipole, is one of the most fundamental multipoles that break not only timereversal but also space-inversion symmetries. The magnetic quadrupoles have attracted growing interest in the study of a linear magnetoelectric (ME) effect ${ }^{9-13}$, nonreciprocal optical responses ${ }^{14}$, and magnetotransport phenomena ${ }^{15}$ as well as their possible relevance to superconductivity ${ }^{16,17}$. By combining the magnetic quadrupoles with chirality, one can expect further exotic phenomena distinct from those arising due to the magnetization and chirality. To our knowledge, however, such phenomena have been much less explored so far.

In this paper, based on symmetry considerations, we propose that magnetic quadrupole of a $Q_{X Y}$ type (magnetic analogue of the $d_{x y}$ electron orbital, see Fig. 1a) and chirality together give rise to unusual induction of magnetization whose direction is orthogonal to an applied magnetic field and reversed by a switching of chirality (see Fig. 1 for a conceptual illustration of this proposal). We substantiate this proposal through measurements of ME effects in an insulating chiral-lattice antiferromagnet $\mathrm{Pb}(\mathrm{TiO})$ $\mathrm{Cu}_{4}\left(\mathrm{PO}_{4}\right)_{4}$ exhibiting a ferroic order of the $Q_{X Y}$ magnetic quadrupoles. Moreover, we demonstrate that this "orthogonal" magnetization allows controlling the sign of $Q_{X Y}$ with a magnetic field only, which is generally impossible in an achiral counterpart. Furthermore, our cluster mean-field calculations successfully reproduce all the experimental observations. The results are also discussed based on a cluster multipole decomposition method and a phenomenological approach.

\section{RESULTS}

Proposal of orthogonal magnetization by combination of magnetic quadrupole and chirality

Before presenting our proposal of orthogonal magnetization, we summarize transformation properties of the following physical quantities crucial in this work: electric polarization (P), magnetization $(\mathbf{M})$, toroidal moment $(\mathbf{T})$ defined as $\mathbf{P} \times \mathbf{M}$, and chirality $(C)$. Here, the parameter $C= \pm 1$ represents the sign of chirality (handedness). The vectors $\mathbf{P}, \mathbf{M}$, and $\mathbf{T}$ are transformed identically under proper rotations, while they are transformed in a different manner under space-inversion, time-reversal, and mirror plane operations. Recently, Hlinka ${ }^{18}$ pointed out that vector-like and director-like physical quantities can be distinguished by their transformations under operations of space-inversion, time-reversal, and mirror plane parallel to the vector (director). Using a notation $\{ \pm, \pm, \pm\}$, where the first, second, and third signs represent the invariance $\{+\}$ or variance $\{-\}$ under these operators, respectively, we can write transformations $\{-,+,+\}$ for $\mathbf{P} ;\{+,-,-\}$ for $\mathbf{M} ;\{-,-,+\}$ for $\mathbf{T}$; and $\{-,+,-\}$ for $C$. One can find that the product of $\mathbf{T}$ and $C$ is transformed identically with $\mathbf{M}$. Hence, $\mathbf{T}$ combined with $C$ can linearly induce $\mathbf{M}$ along parallel/antiparallel to $\mathbf{T}$, as recently discussed in ref. ${ }^{19}$. This is the key piece for our proposal described below. 


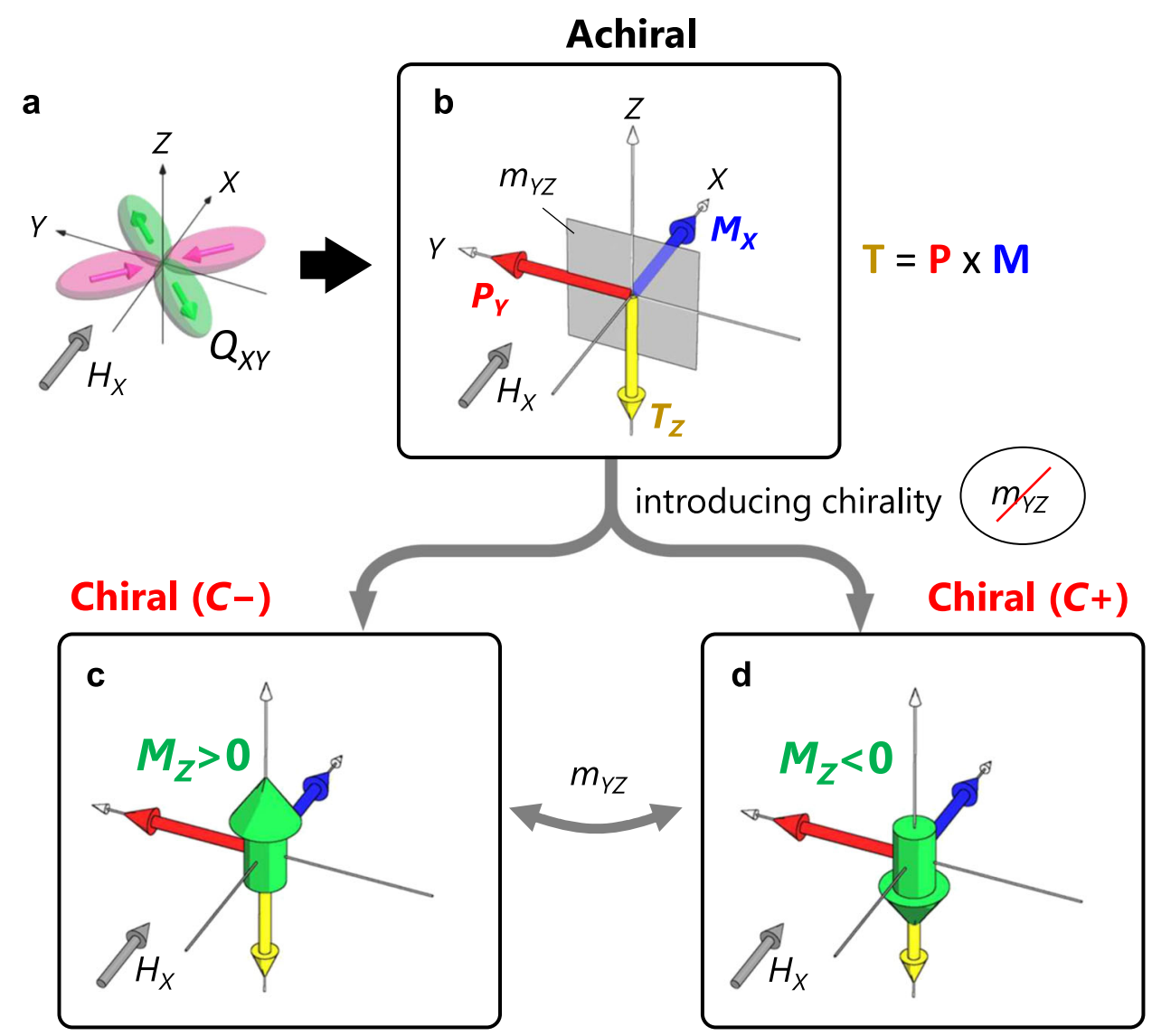

Fig. 1 Conceptual illustration of orthogonal magnetization induced by magnetic quadrupole and chirality. a Schematic illustration of the $Q_{X Y}$-type magnetic quadrupole. In an applied magnetic field along the $X$ axis $\left(H_{X}\right), Q_{X Y}$ produces the electric polarization along the $Y$ axis through the linear magnetoelectric effect. b Directions of $H_{X}$ induced $P_{Y}$, the trivial $H_{X}$ induced magnetization along the $X$ axis $\left(M_{X}\right)$, and the toroidal moment along the $Z$ axis defined by $\mathbf{T}=\mathbf{P} \times \mathbf{M}$. The system is achiral because the mirror plane parallel to the $Y Z$ plane $m_{Y Z}$ is present. c, d A pair of chiral systems $C-$ (c) and $C+(\mathbf{d})$ obtained by breaking the mirror symmetry of $m_{Y Z}$ in the achiral system (b). They are related by $m_{Y Z}$. Combination of $T_{Z}$ and chirality generates the new Z-axis component of the magnetization $\left(M_{Z}\right)$ in an applied $H_{X}$, whose sign is reversed by a chirality switching. This orthogonal magnetization is the subject of this work.

Our proposal is illustrated in Fig. 1. We consider the $Q_{x r}$-type magnetic quadrupole (Fig. 1a) and, first of all, an achiral case. Note that $Q_{X Y}$ itself is achiral because it is invariant under mirror operations such as the one parallel to the $Y Z$ plane $\left(m_{Y Z}\right)$. As a result of breaking of time-reversal and space-inversion symmetries, $Q_{X Y}$ can lead to a linear ME effect $\left(P_{i}=a_{i j} H_{j}\right.$, where $i, j=X, Y$, $Z$ ) with finite ME tensor components, $a_{X Y}=a_{Y X}$. It indicates that an application of a magnetic field $(\mathbf{H})$ along the $X$ axis $\left(H_{X}\right)$ linearly induces $\mathbf{P}$ along the $Y$ axis $\left(P_{Y}\right)$, besides a trivial $\mathbf{M}$ along the $X$ axis $\left(M_{X}\right)\left(H_{X}=X_{X X} M_{X}\right.$, where $X_{X X}$ is the susceptibility). Accordingly, the system in the applied $H_{X}$ possesses the orthogonal configuration of $\mathbf{P}$ and $\mathbf{M}$, and, hence, $\mathbf{T}=\mathbf{P} \times \mathbf{M}$ along the $Z$ axis $\left(T_{Z}={ }_{X X X} a_{Y X} H_{X}{ }^{2}\right)$. The system remains achiral because $m_{Y Z}$ survives (Fig. 1b). Now, we introduce chirality by breaking mirror symmetries. Recalling that $\mathbf{T}$ combined with $C$ can induce $\mathbf{M}$ parallel/unparallel to $\mathbf{T}$, one can expect a new $Z$-axis component of $\mathbf{M}\left(M_{Z}\right)$, which is phenomenologically expressed as

$M_{Z}=\lambda C T_{Z}$

where $\lambda$ is a proportionality constant. Combined with $T_{Z}=$ $X_{X X} a_{Y X} H_{X}^{2}$, Eq. (1) is rewritten as

$M_{Z}=\lambda_{X X X} a_{Y X} C H_{X}^{2}$

This equation predicts that, first of all, the combination of $Q_{X Y}$ and chirality gives rise to the unusual induction of second-order nonlinear magnetization $M_{Z}$ whose direction is orthogonal to the applied $H_{X}$. Moreover, this "orthogonal" magnetization is reversed between the chiral pairs $(C=-1$ and $C=+1)$ related by $m_{Y Z}$ (compare Fig. 1c, d) and is also reversed by a switching of a sign $Q_{X Y}$ that reverses the $a_{Y X}$ sign. In the following, we will examine this proposal.

\section{Crystal chirality, antiferromagnetic state, and in-field states of $\mathrm{Pb}(\mathrm{TiO}) \mathrm{Cu}_{\mathbf{4}}\left(\mathrm{PO}_{4}\right)_{4}$}

Our target antiferromagnet $\mathrm{Pb}(\mathrm{TiO}) \mathrm{Cu}_{4}\left(\mathrm{PO}_{4}\right)_{4}$ (hereafter, abbreviated as $\mathrm{PbTCPO}$ ) has a chiral crystal structure belonging to the space group $P 42_{1} 2^{20}$. It is possible to grow homo-chiral or enantiopure single crystals of both left-handed and right-handed forms, which can be distinguished by a natural optical activity. We define the chirality of levo and dextro rotatory crystals as $C$ - and $\mathrm{C}+$, respectively. The crystal structure of PbTCPO consists of characteristic $\mathrm{Cu}_{4} \mathrm{O}_{12}$ square cupolas, as shown in Fig. $2 \mathrm{a}$, and the unit cell contains one upward and one downward $\mathrm{Cu}_{4} \mathrm{O}_{12}$ square cupola. Here, the $X, Y$, and $Z$ axes are taken parallel to [110], [110], and [001], respectively. Figure $2 \mathrm{~b}, \mathrm{c}$ shows the structures of $C-$ and $C+$ crystals viewed along the $Z$ axis, respectively, in which only $\mathrm{Cu}$ ions are depicted for clarity. In the $\mathrm{C}$ - crystal, the $\mathrm{Cu}_{4}$ square of the upward cupola $\left[\mathrm{Cu}_{4}(\mathrm{U})\right]$ rotates in a counterclockwise direction, while that of the downward cupola $\left[\mathrm{Cu}_{4}(\mathrm{D})\right]$ rotates in a clockwise direction. A mirror operation parallel to the $Y Z$ plane $\left(m_{Y Z}\right)$ mutually converts between the $C+$ and $C$ - crystals. Accordingly, the rotation directions of $\mathrm{Cu}_{4}(\mathrm{U})$ and $\mathrm{Cu}_{4}(\mathrm{D})$ in the $C+$ crystal are opposite to those in the $C$-crystal. For convenience, we define $\varphi$ as a rotation angle for $\mathrm{Cu}_{4}(U)$, such 
a

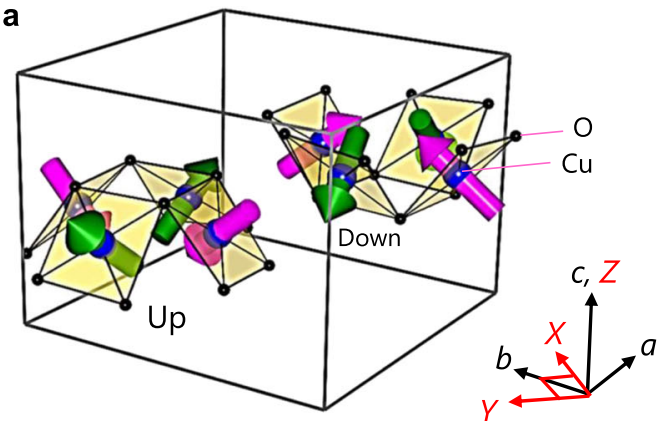

b

b C- crystal c C crystal

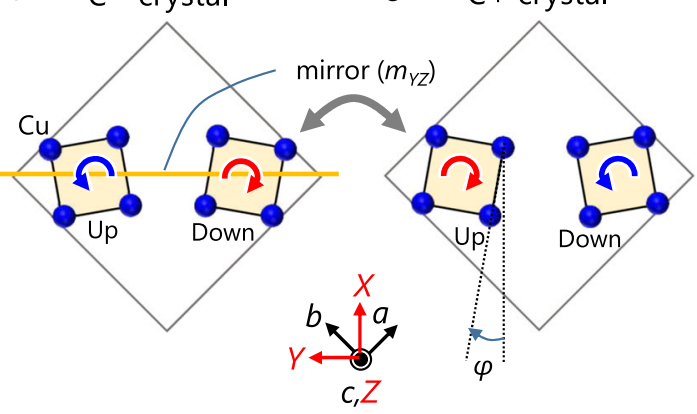

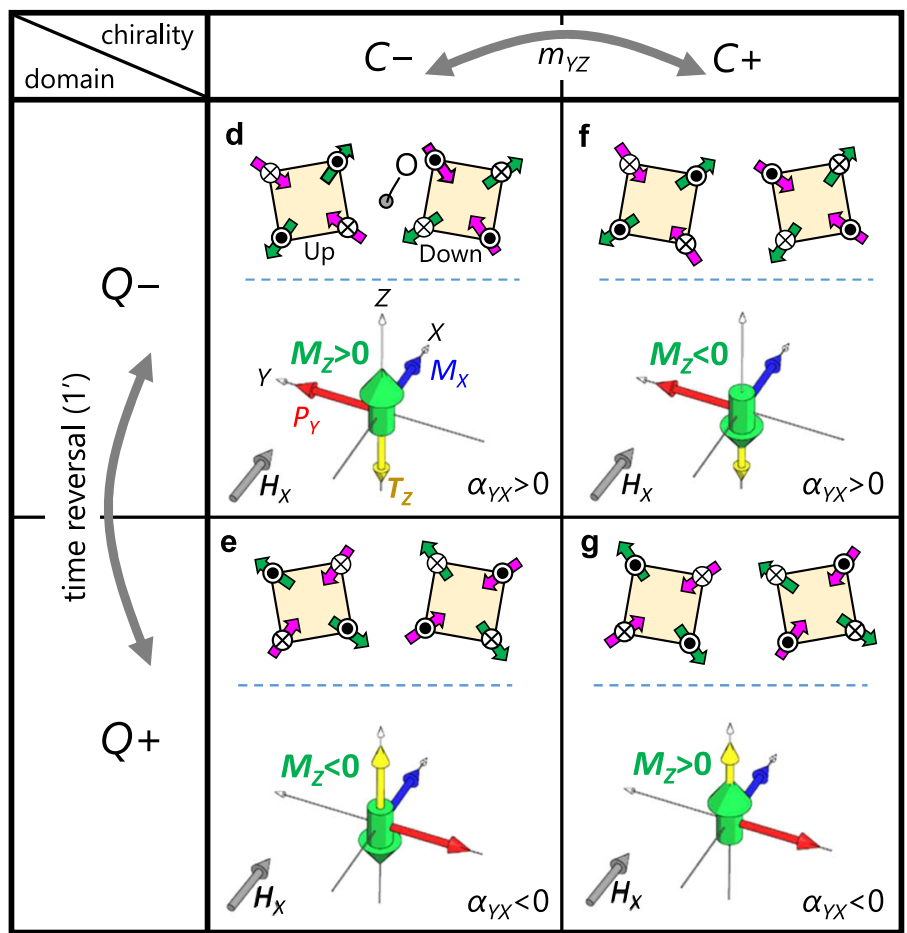

Fig. 2 Crystal structure, chirality, and magnetic domains of $\mathbf{P b}(\mathrm{TiO}) \mathrm{Cu}_{4}\left(\mathrm{PO}_{4}\right)_{4}$. a A three-dimensional view of the crystal and magnetic structures. $\mathrm{Pb}, \mathrm{Ti}$, and $\mathrm{P}$ atoms are omitted for clarity. A unit cell (solid line) contains one upward and one downward $\mathrm{Cu}_{4} \mathrm{O}_{12} \mathrm{square} \mathrm{cupola}$ unit each formed by four $\mathrm{Cu}^{2+}$ ions (large blue balls) and twelve $\mathrm{O}^{2-}$ ligands (black balls). Magenta and green arrows represent inwards and outwards $\mathrm{Cu}^{2+}$ spins, respectively. b, c Staggered rotation of $\mathrm{Cu}_{4}$ squares of upward $\left[\mathrm{Cu}_{4}(\mathrm{U})\right]$ and downward $\left[\mathrm{Cu}_{4}(\mathrm{D})\right]$ units of the $C-(\mathbf{b})$ and $C+\left(\right.$ c) crystals. $\varphi$ represents the rotation angle of $\mathrm{Cu}_{4}(\mathrm{U}) . \varphi>0$ and $\varphi<0$ correspond to $C+$ and $C-$ crystals, respectively. The $C+$ and $C-$ crystal structures are mutually converted by the mirror plane parallel to the $Y Z$ plane $\left(m_{Y Z}\right)$. d-g $Z$-axis views of the magnetic structures of a pair of time-reversed domains $Q+$ and $Q$ - for each chirality. In each panel of $\mathbf{d}-\mathbf{g}$, geometrical configuration of $M_{X}, P_{Y}, M_{Z}$, and $T_{Z}$ induced by an application of a magnetic field along the $X$ axis $\left(H_{X}\right)$ is illustrated. Here, $M_{X}$ and $M_{Z}$ are the magnetization along the $X$ and $Z$ axes, respectively, $P_{Y}$ is the electric polarization along the $Y$ axis, and $T_{Z}$ is the toroidal moment along the $Z$ axis. The sign of the linear magnetoelectric coefficient $a_{Y X}$ that governs the relation of $P_{Y}$ and $H_{X}$ is also indicated.
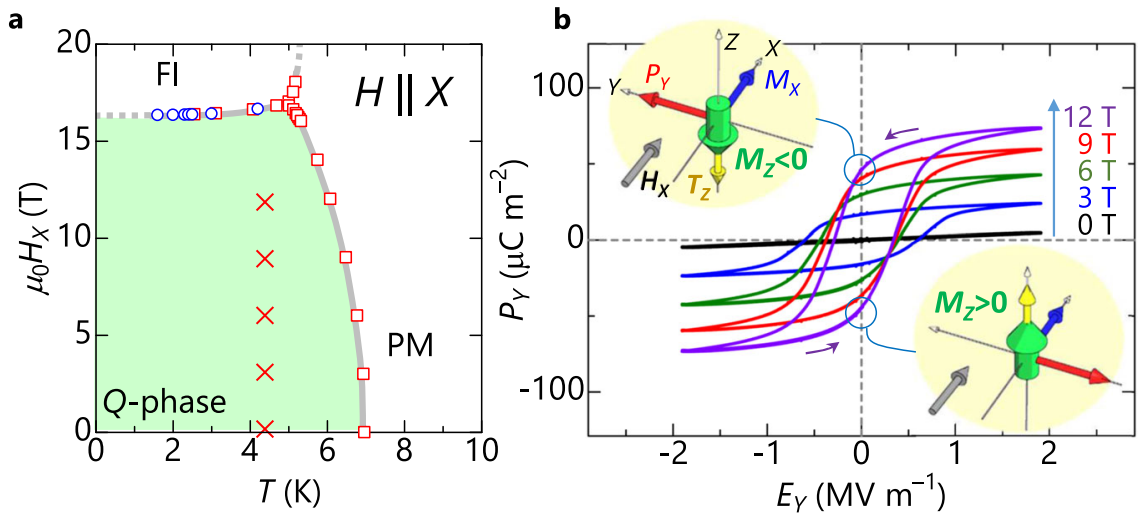

Fig. 3 Fundamental magnetoelectric properties of $\mathbf{P b}(\mathrm{TiO}) \mathrm{Cu}_{\mathbf{4}}\left(\mathrm{PO}_{4}\right)_{4}$. a Temperature $(T$ ) versus magnetic field (H) phase diagram for $\mathbf{H}$ applied along the $X$ axis $\left(H_{X}\right)$. The $Q$-phase represents an antiferromagnetic phase with a magnetic quadrupole order, which is a subject in this work. FI and PM denote field-induced and paramagnetic phases, respectively. b Ferroelectric hysteresis loops at temperature $(T)$ of $4.2 \mathrm{~K}$ measured in $H_{X}$ whose strength is varied between 0 to $12 \mathrm{~T}$ with a $3 \mathrm{~T}$ interval. The electric polarization along the $Y$ axis $\left(P_{Y}\right)$ was measured as a function of the electric field along the $Y$ axis $\left(E_{Y}\right)$. The positions of the measurement temperature and $H_{X}$ are marked by red crosses in the phase diagram in $\mathbf{a}$. Shown in the insets of $\mathbf{b}$ are the geometrical configurations of $H_{X}, P_{Y}, M_{X}, T_{Z}$, and $M_{Z}$ for bistable states at $E_{Y}=0$. Here, $M_{X}$ and $M_{Z}$ are the magnetization along the $X$ and $Z$ axes, respectively, and $T_{Z}$ is the toroidal moment along the $Z$ axis.

that $\varphi>0$ and $\varphi<0$ represent the $C$ - and $C+$ crystals, respectively. According to the structural analysis at room temperature, $|\varphi|$ is estimated to be $4.5^{\circ 20}$.

PbTCPO undergoes an antiferromagnetic transition at $T_{\mathrm{N}}$ $(=7.0 \mathrm{~K} \text { at } 0 \mathrm{~T})^{20}$, as indicated by the magnetic phase diagram in Fig. 3a. In the antiferromagnetic phase, the four Cu spins of each square cupola exhibit a noncoplanar arrangement (Fig. 2a), whose in-plane (Z-plane) and the out-of-plane spin components form two-in two-out and up-down-up-down arrangements, respectively (see Fig. $2 \mathrm{~d}$ ). The magnetic point group of the antiferromagnetic state is $4^{\prime} 22^{\prime}$, which breaks both spaceinversion and time-reversal symmetries, and allows for an ordering of $Q_{X Y}$ and the corresponding linear ME effect with finite $a_{Y X}$ $\left(=a_{Y X}\right)$. As depicted in Fig. $2 \mathrm{~d}-\mathrm{g}$, each chiral crystal can possess a 

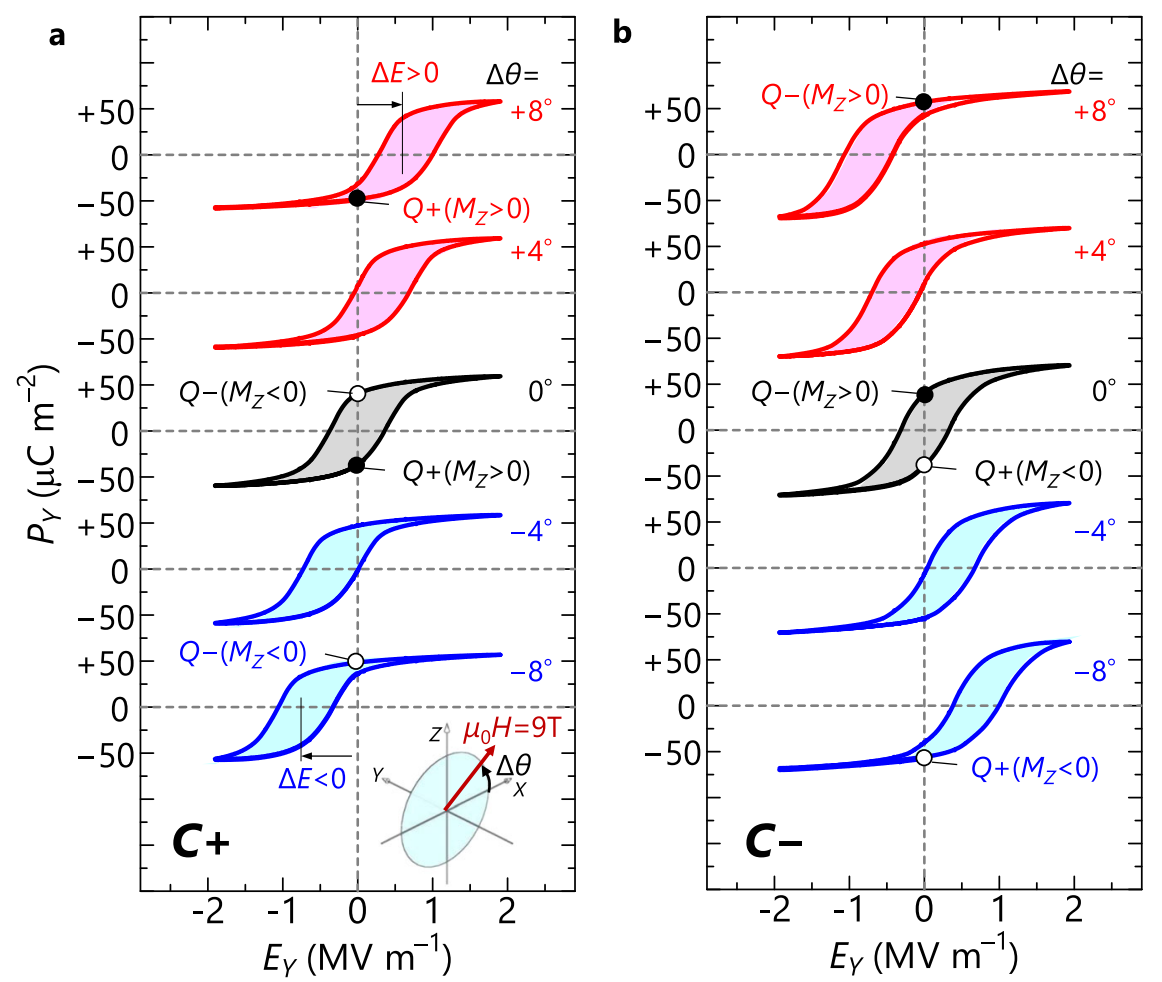

Fig. 4 Chirality-magnetism coupling as probed via ferroelectric hysteresis loops. $\mathbf{a}$, $\mathbf{b} P_{Y} E_{Y}$ hysteresis loops for $\mathbf{a} C+$ and $\mathbf{b} C-\mathrm{crystals}$ in an applied $\mathbf{H}$ tilted from the $+X$ direction by an angle $\Delta \theta$ of $-8^{\circ} \leq \Delta \theta \leq+8^{\circ}$. Definition of $\Delta \theta$ is illustrated in the inset of a. In the finite $\mathbf{H}$ tilting, the ferroelectric hysteresis loops are shifted along the $E_{Y}$ axis by $\Delta E$, whose sign depends on the sign of $\Delta \theta$ as well as the chirality switching. The magnetic quadrupole domains $(Q+/ Q-)$ stabilized by the tilted $\mathbf{H}$ are indicated by the white and black circles, together with respective signs of orthogonal magnetization $M_{Z}$.

pair of magnetic quadrupole domains, $Q-\left(Q_{X Y}<0\right)$ and $Q+\left(Q_{X Y}>\right.$ $0)$, with opposite signs of $a_{Y X}$, which are mutually converted by time-reversal operation ( $\left.1^{\prime}\right)$ [compare Fig. $2 \mathrm{~d}(2 \mathrm{f})$ and $2 \mathrm{e}(2 \mathrm{~g})$ ]. (The sign of $Q_{X Y}$ is directly calculated based on the magnetic structure; see Supplementary Figs. 1 and 2, and Supplementary Note 1) According to the previous theoretical analysis of the ME coupling in $\mathrm{PbTCPO}^{21}$, the signs of $a_{Y X}$ are negative and positive for the $Q+$ and $Q$-domains, respectively, while they remain unchanged under the chirality switching. Because the linear $M E$ effect $P_{Y}=$ $a_{Y X} H_{X}$ was experimentally confirmed ${ }^{20}$, PbTCPO realizes the aforementioned situation depicted in Fig. 1, where the anomalous induction of second-order nonlinear magnetization $M_{Z}$ by applying $H_{X}$ can be anticipated. (This is the lowest-order induction of $M_{Z}$ by $H_{X}$ because a linear induction of $M_{Z}$ by $H_{X}$ is prohibited by the $4^{\prime} 22^{\prime}$ symmetry ${ }^{22,23}$ ). Recalling that $M_{Z}$ is reversed by a switching of chirality as well as a switching of $Q_{X Y}$ [Eq. (2)], we explicitly indicate in Fig. $2 \mathrm{~d}-\mathrm{g}$ the sign of $M_{Z}$ for the four distinct states, together with the configurations of $P_{Y}, M_{X}$, and $T_{Z}$. We assume $\lambda$ is positive for the sign of $M_{Z}$ to be consistent with experimental results, which will be described below.

\section{Magnetic-field-induced ferroelectric hysteresis loops}

To examine the proposed orthogonal magnetization $M_{Z}$ in PbTCPO, we perform measurements of $P_{Y}$ in various conditions in terms of external $\mathbf{E}$ and $\mathbf{H}$, since the sign of $P_{Y}$ in finite $H_{X}$ directly probes the sign of $M_{Z}$ (see Fig. $2 \mathrm{~d}-\mathrm{g}$ ). Figure $3 \mathrm{~b}$ shows $E_{Y}$ dependence of $P_{Y}$ for a $C+$ crystal measured at $4.2 \mathrm{~K}\left(<T_{N}\right)$ in an applied $H_{X}$. The strength of $\mu_{0} H_{X}$ was varied between 0 and $12 \mathrm{~T}$ with a $3 \mathrm{~T}$ interval (red crosses in the phase diagram of Fig. 3a). As seen in Fig. 3b, a finite spontaneous $P_{Y}\left(P_{Y}\right.$ at $\left.E_{Y}=0\right)$ emerges in the finite $H_{X}$, whose magnitude increases almost linearly with increasing $H_{X}$, indicating that the spontaneous $P_{Y}$ originates from $P_{Y}=a_{Y X} H_{X}$. Moreover, we observe a clear ferroelectric hysteresis loop, a reversal of $P_{Y}$ at a certain threshold magnitude of $E_{Y}$. This reversal corresponds to the switching between the $Q-$ and $Q+$ domains with opposite signs of $M_{z}$ as depicted in the inset of Fig. 3b.

A signature for the existence of $M_{Z}$ is observed when the applied $\mathbf{H}$ is slightly tilted from the $X$ axis to the $Z$ axis by an angle $\Delta \theta$, so that a small $Z$-axis component of the magnetic field $\left(H_{Z}\right)$ is present (see the inset of Fig. 4a). Figure 4a displays the ferroelectric hysteresis loops for the $C+$ crystal measured at $4.2 \mathrm{~K}$ in the tilted $\mathbf{H}$ of $\mu_{0} H=9 \mathrm{~T}\left(-8^{\circ} \leq \Delta \theta \leq+8^{\circ}\right)$. It is seen that introducing the finite $H_{Z}(\Delta \theta \neq 0)$ causes a significant shift of the center of the ferroelectric hysteresis loops along the $E_{Y}$ axis, or a bias effect. Moreover, the bias electric field $(\Delta E)$ is positive for $H_{Z}>$ $0(\Delta \theta>0)$, whereas negative for $H_{Z}<0(\Delta \theta<0)$. This means that the original degeneracy between the $Q-$ and $Q+$ domains at $E_{Y}=$ 0 is broken by the small $H_{Z}$ component; $H_{Z}>0$ stabilizes the $Q+$ domain $\left(P_{Y}<0\right.$, Fig. $\left.2 \mathrm{~g}\right)$, whereas $\mathrm{H}_{Z}<0$ stabilizes the $Q-$ domain $\left(P_{Y}>0\right.$, Fig. 2f). Assuming that the $Q+$ and $Q-$ domains have $M_{Z}>$ 0 and $M_{Z}<0$, respectively (i.e., proportionality constant $\lambda>0$ ), the interaction of $H_{Z}$ and $M_{Z}\left(=-H_{Z} M_{Z}\right)$ should differentiate the free energy of the $Q$ - and $Q+$ domains by $2 H_{Z} M_{Z}$, which naturally explains the observed domain stabilization. This provides the first evidence for the existence of $M_{Z}$.

Second, and conclusive evidence for the existence of $M_{Z}$ is observed in results of the same measurements for a crystal with the opposite chirality ( $C$-crystal). As shown in Fig. $4 \mathrm{~b}$, the sign of $\Delta E$ for the $C$ - crystal is completely reversed from that of the $C+$ crystal, meaning that the quadrupole domain stabilized in the same condition of $H_{z}$ is opposite between the $C-$ and $C+$ crystals. This is reasonably explained by assuming that the signs of $M_{Z}$ of the respective quadrupole domains are reversed by the chirality switching, in complete agreement with Eq. (2). Therefore, our chirality-specific measurements of the ferroelectric hysteresis 
loops in the tilted $\mathbf{H}$ successfully demonstrate the existence of the proposed $M_{Z}$ arising from the combination of $Q_{X Y}$ and chirality.

We also confirm that the sign of $\Delta E$ does not alter by a reversal of the applied $\mathbf{H}$ (i.e., $180^{\circ}$ rotation in the $Z X$ plane), as shown in Supplementary Fig. 3 . This is consistent with an expected feature of our proposal, as explained below. Let us consider the $C+$ crystal and a reversal of $\mathbf{H}$ from $H_{X}>0$ and $H_{Z}>0$ to $H_{X}<0$ and $H_{Z}<0$. As already depicted in Fig. $4 a$, before the $\mathbf{H}$ reversal $\left(H_{X}>0, H_{Z}>0\right)$, the $Q+$ domain with $P_{Y}<0$ and $M_{Z}>0$ is stabilized. This is reflected as the positive $\Delta E$ in the ferroelectric hysteresis loops. After the reversal of $\mathbf{H}\left(H_{X}<0, H_{Z}<0\right)$, due to the second-order dependence of $M_{Z}$ to $H_{X}$ [Eq. (2)], the $Q+$ and $Q-$ domains keep in having $M_{Z}>0$ and $M_{Z}<0$, respectively. Thus, the interaction $-H_{Z} M_{Z}$ will stabilize the $Q-$ domain with $M_{Z}<0$. In the applied $H_{X}<0$, the $Q$ - domain $\left(a_{Y X}>0\right.$, see Fig. 2) has $P_{Y}<0$. To make the $P_{Y}<0$ state stable at $E_{Y}=0, \Delta E$ will be positive, which is thus identical with that before the $\mathbf{H}$ reversal. This $H$-reversalindependent behavior is completely different from a previously reported biased hysteresis loop in a ferroelectric ferromagnet $\mathrm{Dy}_{0.7} \mathrm{~Tb}_{0.3} \mathrm{FeO}_{3}$, where $\Delta E$ depends linearly on an applied $\mathbf{H}^{24}$. A more detailed analysis of the observed bias effect in the present compound PbTCPO is given in Supplementary Fig. 4 and Supplementary Note 2.

\section{Single domain state of magnetic quadrupole by tilted magnetic field}

The breaking of the degeneracy of the $Q+$ and $Q$ - domains in tilted $\mathbf{H}$ raises an intriguing possibility that a single domain state is achieved by cooling a sample across $T_{N}$ with $\mathbf{H}$ only. Figure 5 shows the temperature dependence of $P_{Y}$ for the $C+$ crystal in $\mu_{0} H=9 \mathrm{~T}$ with $\Delta \theta=0, \pm 4$, or $\pm 8^{\circ}$ (see the inset), measured on warming without any electric field. No electric field was applied during a cooling process before each measurement. At $\Delta \theta=0, P_{Y}$ below $T_{N}$ is vanishingly small. This indicates that the $Q+$ and $Q-$ domains coexist in the sample, in agreement with the degeneracy of the two domains in $H_{Z}=0$. By contrast, at $\Delta \theta=+8^{\circ}\left(H_{Z}>0\right)$ and $\Delta \theta=-8^{\circ}\left(H_{Z}<0\right)$, finite $P_{Y}$ of opposite signs is observed. The values of $+40 \mu \mathrm{C} \mathrm{m}^{-2}\left(-40 \mu \mathrm{C} \mathrm{m}^{-2}\right)$ at $4.2 \mathrm{~K}$ are close to those corresponding to the single domain state of $Q+(Q-)$ evaluated as the spontaneous $P_{Y}$ in the hysteresis loops (see Fig. 4a). Thus, the single domain states of $Q+$ and $Q$ - are successfully formed by tilted $\mathbf{H}$ with $H_{Z}>0$ and $H_{Z}<0$, respectively. This result is remarkable, because achievement of a single domain state in ME antiferromagnets generally requires not only $\mathbf{H}$, but also $\mathbf{E}$. Therefore, the orthogonal magnetization provides the distinct way of controlling ME antiferromagnetic domains free from $\mathbf{E}$.

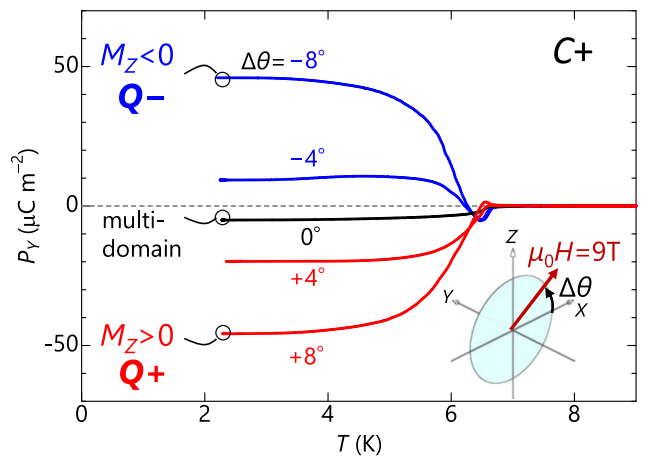

Fig. 5 Temperature dependence of $P_{Y}$ for the $C+$ crystal in an applied $\mu_{0} H$ of $9 \mathrm{~T}$ with $\Delta \theta=0, \pm 4^{\circ}$, and $\pm 8^{\circ}$. No electric field was applied during each measurement as well as during a cooling process before each measurement.

\section{Cluster mean-field calculations}

The validity of the chirality-induced orthogonal magnetization is further examined theoretically by the cluster mean-field calculations of nonlinear magnetization. According to the previous studies $^{21,25}$, overall magnetic and ME behaviors of PbTCPO are well reproduced by an effective spin-1/2 model which is described as

$$
\begin{aligned}
H_{\text {eff }}= & \sum_{\langle i, j\rangle}\left[J_{1} \mathbf{S}_{i} \cdot \mathbf{S}_{j}-\mathbf{D}_{i j} \cdot\left(\mathbf{S}_{i} \times \mathbf{S}_{j}\right)\right]+J_{2} \sum_{\langle\langle i, j\rangle\rangle} \mathbf{S}_{i} \cdot \mathbf{S}_{j} \\
& +J^{\prime} \sum_{(i, j)} \mathbf{S}_{i} \cdot \mathbf{S}_{j}+J^{\prime \prime} \sum_{([i, j])} \mathbf{S}_{i} \cdot \mathbf{S}_{j}-g \mu_{B} \sum_{i} \mathbf{H} \cdot \mathbf{S}_{i} .
\end{aligned}
$$

Here, $J_{1}$ and $J_{2}$ are the nearest-neighbor and next-nearest-neighbor intracupola symmetric exchange interactions, respectively, while $J^{\prime}$ and $J^{\prime \prime}$ are the intralayer- and interlayer-intercupola interactions, respectively (see Supplementary Fig. 5). $\mathbf{D}_{i j}$ is the so-called Dzyaloshinskii-Moriya (DM) vector describing an antisymmetric DM interaction at $J_{1}$ bonds that reflects the geometry of the $\mathrm{Cu}_{4} \mathrm{O}_{12}$ unit. As depicted in Fig. $6 a$, the direction of $\mathbf{D}_{i j}$ is largely tilted from the $Z$ axis to the $X$ or $Y$ axis by $80^{\circ}$. In the previous calculations, the arrangement of $\mathbf{D}_{i j}$ at different $J_{1}$ bonds was assumed so as to preserve the mirror symmetry $m_{Y Z}$ (see Fig. 6a); in other words, the effect of the chirality was neglected. In the present calculation, by contrast, the effects of the chirality are considered by introducing a staggered rotation of $\mathbf{D}_{i j}$ about the $Z$ axis by an angle $\varphi_{\mathrm{DM}}=5^{\circ}$, which is accompanied by the staggered rotation of $\mathrm{Cu}_{4}$ squares by $\varphi=5^{\circ}$ (corresponding to $C+$ crystal), as shown in Fig. $6 \mathrm{~b}$. We shall call this model the chiral spin-1/2 model. Except for the rotation of $\mathbf{D}_{i j}$, we use the same set of parameters as in the previous calculations: $J_{1}=1, J_{2}=1 / 7, J^{\prime}=3 / 4, J^{\prime \prime}=1 / 100$, and $D=\left|\mathbf{D}_{i j}\right|=1.1$.

First, we have calculated the magnetization along the $Z$ axis, $m_{Z \text {, }}$ for the two magnetic domains $q+\left(q_{X Y}>0\right)$ and $q-\left(q_{X Y}<0\right)$, in the case of $\varphi_{\mathrm{DM}}=0$ and $5^{\circ}$. A magnetic field $\left(\mathbf{h}=g \mu_{\mathrm{B}} \mathbf{H}\right)$ along the $X$ axis, $h_{X}$, is varied between -1.5 and 1.5 , while $\mathbf{h}$ along the $Z$ axis, $h_{Z}$ is fixed at zero. (We use the lowercase symbols $q, q_{X Y}, \mathbf{h}$, and $m$ to distinguish them from the respective uppercase symbols for the experiment.) The result in both cases of $\varphi_{\text {DM }}$ is shown in Fig. $6 \mathrm{c}$. It is seen that, when the chirality is neglected (i.e., $\varphi_{\mathrm{DM}}=0$ ), $m_{X}$ is zero within the numerical accuracy for both the $q+$ and $q-$ states. By contrast, when the chirality is introduced (i.e., $\varphi_{\mathrm{DM}}=5^{\circ}$ ), the $q+$ and $q$-states have a finite $m_{Z}$ with the same magnitude but opposite signs. This is consistent with the experimental results. Next, we examine the effect of the field tilting in the $X Z$ plane on the stability of the domains in the chiral system. Figure $6 \mathrm{~d}$ shows the calculated free energy $f$ as a function of the applied $h_{z}$ at a fixed $h_{X}$ of 0.5 . It is found that the original degeneracy between the $q+$ and $q$ - states at $h_{z}=0$ is lifted by introducing finite $h_{z}$ in good agreement with the experimental results. Moreover, the relationship between the stabilized domain and the sign of $h_{z}$ ( $q+$ by $h_{z}>0$ and $q-$ by $h_{z}<0$, respectively) is also consistent with the experimental results (see Fig. $2 f, g$ ). Taken together, our chiral spin-1/2 model successfully reproduces all the experimental results, strongly supporting the existence of the chirality-induced orthogonal magnetization.

\section{Cluster multipole analysis and phenomenological approach}

Motivated by the recent development of the cluster multipole theory, we analyze the experimental results based on the cluster multipoles. It is indicated by Eq. (2) that the orthogonal magnetization $M_{Z}$ is categorized as a second-order nonlinear magnetization with the general form of $M_{i}^{(2)}=X_{i j k} H_{j} H_{k}(i, j, k=X, Y, Z)$. $X_{i j k}$ is the second-order nonlinear magnetic susceptibility and the component $X_{Z X X}$ corresponds to $\lambda_{X x x} a_{Y X} C$ of Eq. (2). According to the comprehensive theoretical work by Hayami et al. ${ }^{7}, M_{i}^{(2)}$ is associated with magnetic octupoles and/or magnetic toroidal quadrupoles (Supplementary Note 1). As described in Supplementary Fig. 6 and Supplementary Note 3, our additional calculations based on the chiral spin-1/2 model successfully 

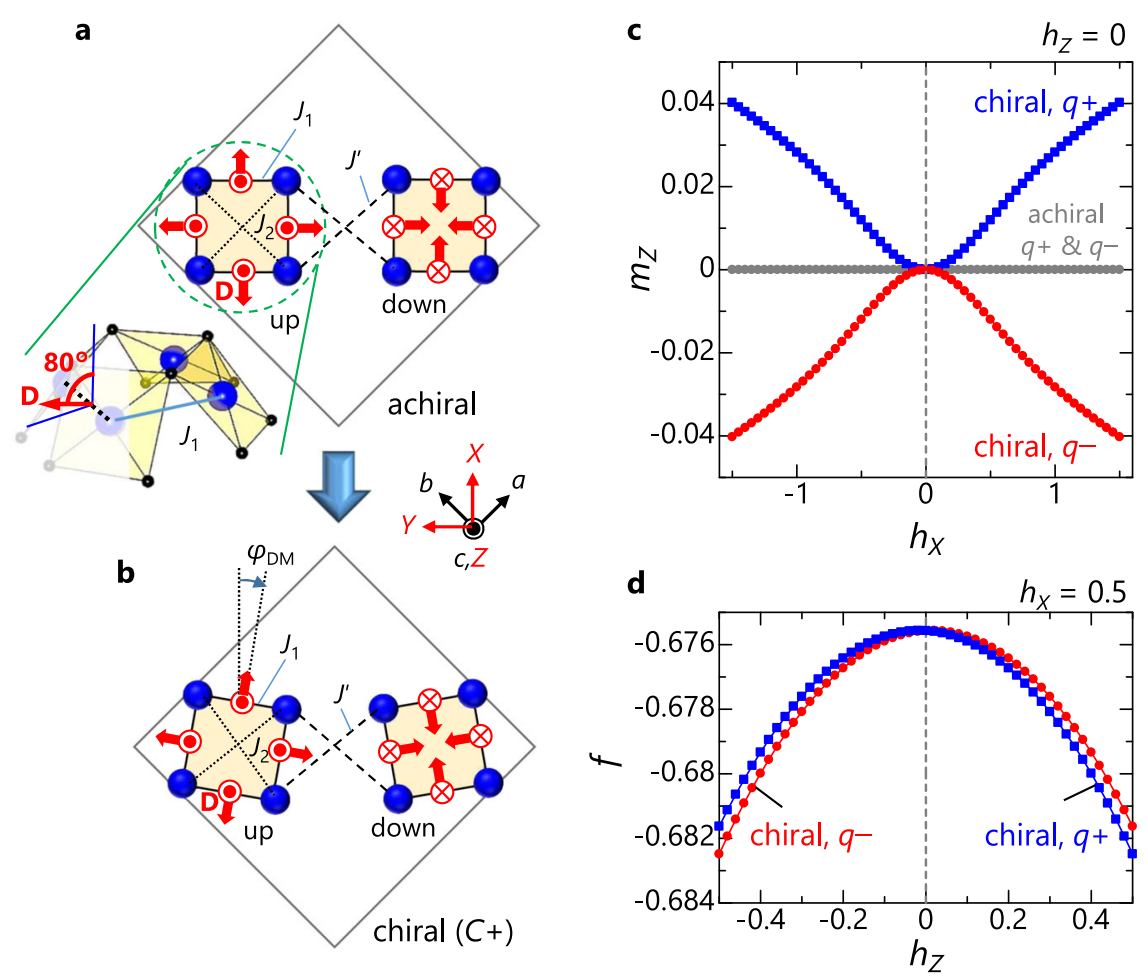

Fig. 6 Calculations based on the chiral spin-1/2 model. a, b Achiral (a) and chiral spin 1/2 model (b) and parameters setting. The red thick arrows represent the DM vector at the $J_{1}$ bond, $\mathbf{D}$, which is considerably tilted from the $Z$ axis by $80^{\circ}$. A staggered rotation of $\mathbf{D}$ by $\varphi_{\mathrm{DM}}=5^{\circ}$ is introduced in the chiral spin-1/2 model. c Calculated results of the magnetization along the $Z$ axis, $m_{Z}$, as a function of magnetic field (h) along the $X$ axis, $h_{X}$, in the absence of $\mathbf{h}$ along the $Z$ axis $\left(h_{Z}\right)$. The gray symbols represent $m_{Z}$ in the case of $\varphi_{\text {DM }}=0$ for both $q+$ and $q-$, which are zero within the numerical accuracy. The blue squares and the red circles denote $m_{Z}$ for $q+$ and $q-$, respectively, in the case of $\varphi_{\mathrm{DM}}=5^{\circ}$. $\mathbf{d} h_{Z}$ dependence of the free energy $f$ at $h_{X}=0.5$ in the case of $\varphi_{\mathrm{DM}}=5^{\circ}$.

identify that an $O_{Z}^{\beta}$-type magnetic octupole (see Fig. 7 for schematic representation) gives a dominant contribution to $X_{z x x}$. The $O_{Z}^{\beta}$ is expressed as

$$
O_{Z}^{\beta}=\sum_{i} \sqrt{15}\left[\frac{1}{2}\left(X_{i}^{2}-Y_{i}^{2}\right) m_{i}^{Z}+Z_{i}\left(X_{i} m_{i}^{X}-Y_{i} m_{i}^{Y}\right)\right]
$$

where the sum is taken over eight $\mathrm{Cu}$ ions in the unit cell and $m_{i}^{n}$ represents an $n$-axis $(n=X, Y, Z)$ component of a magnetic dipole $\mathbf{m}_{i}$ of the $i$-th $(i=1-8) \mathrm{Cu}$ ion located at a position $\mathbf{r}_{i}=\left(X_{i}, Y_{i}, Z_{i}\right)$. The origin of the coordinate is taken as the center of the unit cell, which is denoted by "O" in Fig. 2d. We emphasize that the presence of chirality is indispensable for finite $O_{Z}^{\beta}$ in PbTCPO, because a hypothetical magnetic structure (magnetic point group $4^{\prime} / \mathrm{m}^{\prime} \mathrm{mm}^{\prime}$ ) has a PT symmetry $\left(\overline{1}^{\prime}\right)$, which makes $O_{Z}^{\beta}$ strictly zero (see Supplementary Fig. 1 and Supplementary Note 1).

Figure 7 conceptually illustrates a coupling among $O_{Z}^{\beta}, Q_{X Y}$, and the chirality $C$, as well as field responses of $O_{Z}^{\beta}$ and $Q_{X Y}$, which is obtained by a phenomenological approach based on the Landau theory ${ }^{26,27}$ as described below. To take chirality into account, we use a hypothetical achiral crystal structure with the space group $\mathrm{P} 4 / \mathrm{nmm}$, which was recently observed in a sister compound $\mathrm{Ba}$ (TiO) $\mathrm{Cu}_{4}\left(\mathrm{PO}_{4}\right)_{4}$ at high temperatures ${ }^{28}$. Table 1 summarizes the transformations of various order parameters for PbTCPO based on the generators of the point group $4 / \mathrm{mmm}^{22,23,29}$ for the achiral case. We consider this point group, because all the listed parameters are invariant under the translational symmetry of $\mathrm{P4} /$ $\mathrm{nmm}$. We also add the time-reversal operation $1^{\prime}$.

From Table 1, one can find that the invariant $E_{1} \sim\left(P_{X} M_{Y}+P_{Y} M_{X}\right)$ $Q_{X Y}$ stands for the achiral case $(4 / \mathrm{mmm})$ [and of course also for the chiral case (422) because 422 is a subgroup of $4 / \mathrm{mmm}$ ]. If we use an electric field $\mathbf{E}$ and a magnetic field $\mathbf{H}$ to manipulate $\mathbf{P}$ and $\mathbf{M}$, we can reformulate $E_{1} \sim\left(E_{X} H_{Y}+E_{Y} H_{X}\right) Q_{X Y}$. This can explain the reason why the sign of $Q_{X Y}$ is controllable by, e.g., $E_{Y} H_{X}$, and is in one-to-one relation with the sign of $a_{Y X}=a_{X Y}\left(=-\partial^{2} E_{1} / \partial E_{X} \partial H_{Y} \propto\right.$ $\left.Q_{X Y}\right)$. Similarly, the invariant $E_{2} \sim\left(H_{Z} H_{X} H_{X}-H_{Z} H_{Y} H_{Y}\right) O_{Z}^{\beta}$ suggests that the sign of $O_{Z}^{\beta}$ is controllable by, i.e., $H_{Z} H_{X} H_{X}$ and is in one-toone relation with the sign of $X_{Z X X}=-X_{Z Y Y}\left(=-\partial^{3} E_{2} / \partial H_{Z} \partial H_{X} \partial H_{X} \propto\right.$ $O_{Z}^{\beta}$ ). Note that the achiral case (i.e., with $m_{X Y}$ ) can only support $Q_{X Y}$ or $O_{Z}^{\beta}$, but not the both, because they transform in opposite ways with respect to $m_{X Y}$. In the experiment, $Q_{X Y}$ was selected for the achiral case. Conversely, $O_{Z}^{\beta}$ (and corresponding $X_{z x x}$ ) was absent for the achiral case.

By contrast, when introducing chirality (i.e., removing $m_{Y Z}$ ), both $Q_{X Y}$ and $O_{Z}^{\beta}$ are transformed identically. Hence, they can coexist, and, moreover, the switching of the sign of $Q_{X Y}$ simultaneously changes the sign of $O_{Z}^{\beta}$. This allows for the cross-control of $Q_{X Y}$ and $O_{Z}^{\beta}$ by their non-conjugate fields, that is, the control of $Q_{X Y}$ by the tilted magnetic field $\left(H_{Z} H_{x} H_{X}\right)$ and the control of $O_{Z}^{\beta}$ by $E_{Y} H_{X}$, as indicated by thick red and blue arrows in Fig. 7b, c. This explains the reason why the quadrupole domains can be controlled by the tilted $\mathbf{H}$. Furthermore, the switching of chirality reverses the sign relation between $Q_{X Y}$ and $O_{Z}^{\beta}$ (compare Fig. 7b,c), because $m_{X Y}$ transforms $Q_{X Y}$ and $O_{Z}^{\beta}$ in opposite ways. It results in a reversal of the sign of $Q_{X Y}$ selected by the tilted $\mathbf{H}$, in agreement with the experimental results. Therefore, all the experimental results originate from the combination among magnetic quadrupoles, magnetic octupoles, and chirality.

\section{DISCUSSION}

In summary, starting from the simple symmetry considerations depicted in Fig. 1, we have proposed that the $Q_{X Y}$ magnetic quadrupole and the chirality together give rise to the anomalous induction of orthogonal magnetization $M_{Z}$ in an applied magnetic field $H_{X}$, which arises from the coupling of the chirality and the 


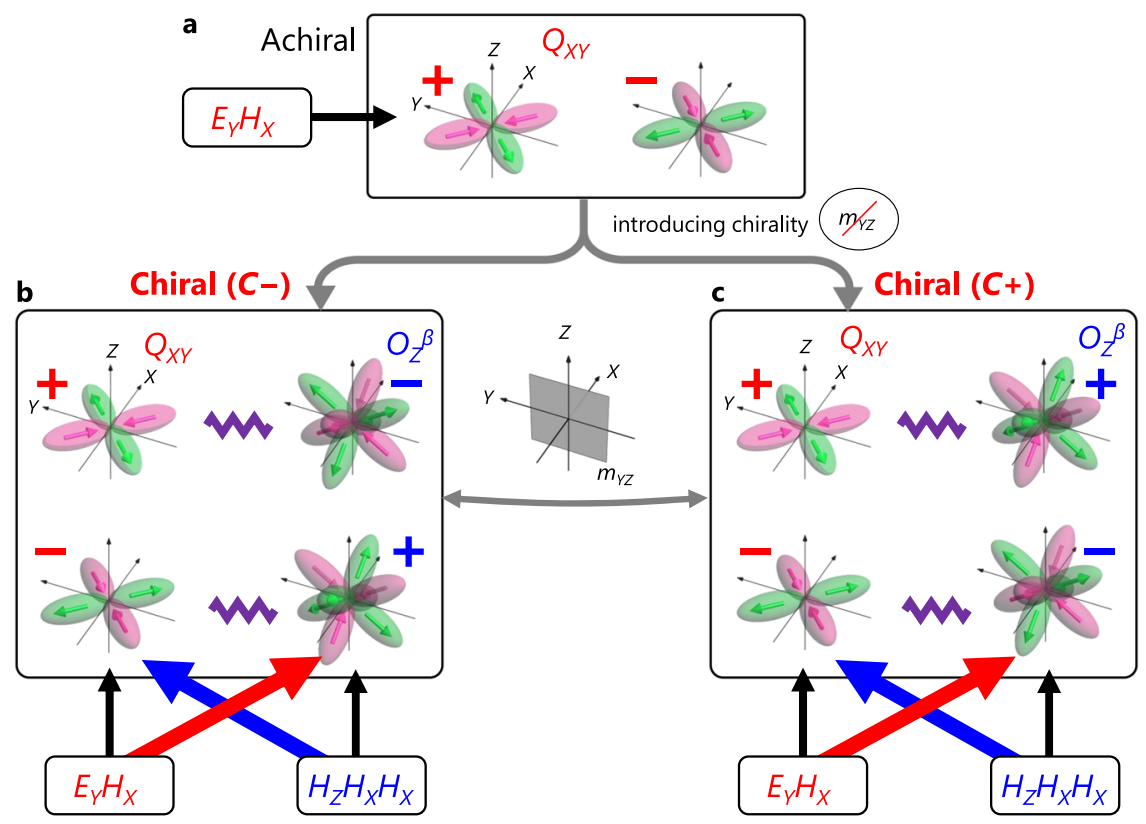

Fig. 7 Conceptual illustration of cross-control of magnetic quadrupole and octupole. a In the case of a hypothetical achiral structure, the antiferromagnetic phase of $\mathrm{Pb}(\mathrm{TiO}) \mathrm{Cu}_{4}\left(\mathrm{PO}_{4}\right)_{4}$ would possess only magnetic quadrupoles $Q_{X Y}$. + and - represent the signs of $Q_{X Y}$. The control of the sign of $Q_{X Y}$ requires a simultaneous application of electric field $E_{Y}$ and magnetic field $H_{X}\left(E_{Y} H_{X}\right)$. $\mathbf{b}$, c By introducing the chirality of the crystal structure, which corresponds to the actual structure of $\mathrm{Pb}(\mathrm{TiO}) \mathrm{Cu}_{4}\left(\mathrm{PO}_{4}\right)_{4}$, the magnetic structure acquires additional octupole moments $O_{Z}^{\beta}$. Here, a pair of chiral structures $C-(\mathbf{b})$ and $C+(\mathbf{c})$ are related to each other by mirror operation $m_{Y Z}$. The signs of $O_{Z}^{\beta}$ depend on those of $Q_{X Y}$. Thus, a switching of the sign of $Q_{X Y}$ accompanies that of $O_{Z}^{\beta}$ or vice versa (see text for details). The signs of $Q_{X Y}$ are controllable by a nonlinear magnetic field $H_{Z} H_{X} H_{X}$. Accordingly, introducing the chirality allows for an unusual cross-control of $Q_{X Y}$ and $O_{Z}^{\beta}$ (represented by thick red and blue arrows); namely, control of $Q_{X Y}$ with a magnetic field only and control of $O_{Z}^{\beta}$ with an electric field in a bias magnetic field. The sign of $Q_{X Y}$ and $O_{Z}^{\beta}$ selected by this cross-control is reversed by a switching of the chirality, as experimentally observed.

Table 1. Transformations of various order parameters for $\mathrm{Pb}(\mathrm{TiO})$ $\mathrm{Cu}_{4}\left(\mathrm{PO}_{4}\right)_{4}$ based on the generators of $4 / \mathrm{mmm}$ point group.

\begin{tabular}{llllll}
\hline Order parameter & $4_{Z}$ & $2_{X}$ & $2_{Y}$ & $m_{Y Z}$ & $1^{\prime}$ \\
\hline$Q_{X Y}$ & $-Q_{X Y}$ & $-Q_{X Y}$ & $-Q_{X Y}$ & $Q_{X Y}$ & $-Q_{X Y}$ \\
$O_{Z}^{\beta}$ & $-O_{Z}^{\beta}$ & $-O_{Z}^{\beta}$ & $-O_{Z}^{\beta}$ & $-O_{Z}^{\beta}$ & $-O_{Z}^{\beta}$ \\
$P_{X}$ & $P_{Y}$ & $P_{X}$ & $-P_{X}$ & $-P_{X}$ & $P_{X}$ \\
$P_{Y}$ & $-P_{X}$ & $-P_{Y}$ & $P_{Y}$ & $P_{Y}$ & $P_{Y}$ \\
$P_{Z}$ & $P_{Z}$ & $-P_{Z}$ & $-P_{Z}$ & $P_{Z}$ & $P_{Z}$ \\
$M_{X}$ & $M_{Y}$ & $M_{X}$ & $-M_{X}$ & $M_{X}$ & $-M_{X}$ \\
$M_{Y}$ & $-M_{X}$ & $-M_{Y}$ & $M_{Y}$ & $-M_{Y}$ & $-M_{Y}$ \\
$M_{Z}$ & $M_{Z}$ & $-M_{Z}$ & $-M_{Z}$ & $-M_{Z}$ & $-M_{Z}$ \\
\hline
\end{tabular}

We also add the time-reversal operation $1^{\prime}$. The $P_{X}, P_{Y}$, and $P_{Z}\left(M_{X}, M_{Y}\right.$, and $M_{Z}$ ) denote the electric polarization (magnetization) along the $X, Y$, and $Z$ directions, respectively. The generators except for the $m_{Y Z}$ operator generate the group elements for 422 point group (plus time-reversal operation 1).

$H_{X}$-induced toroidal moment $\left(T_{Z}\right)$ due to the linear ME effect of $Q_{X Y}$. Using the model material PbTCPO, we have substantiated this proposal by observing that the domain state of $Q_{X Y}$ can be controlled not only by the electric and magnetic fields together $\left(E_{Y} H_{X}\right)$, but also by the tilted magnetic field only $\left(H_{Z} H_{X} H_{X}\right)$. We have also associated the experimental results with the cluster multipoles, revealing that $M_{Z}$ is viewed as the second-order nonlinear magnetization and originates from the magnetic octupole $O_{Z}^{\beta}$. Moreover, our phenomenological model has revealed that the signs of $Q_{X Y}$ and $O_{Z}^{\beta}$ are coupled due to the chirality, which allows for the unusual cross-control of $Q_{X Y}$ and $O_{Z}^{\beta}$ by the $E_{Y} H_{X}$ and $H_{Z} H_{X} H_{X}$ fields, respectively.

Our observation of the electric-field-free control of the magnetic quadrupole domains in the chiral-lattice antiferromagnet PbTCPO has various implications for other antiferromagnets with ME multipoles (i.e., magnetic monopole, magnetic quadrupole, and magnetic toroidal dipole). Of the 122 magnetic point groups, there are six enantiomorphic, antiferromagnetic groups [222, 422, 4'22' (present case), 32, 622, and 23] that allow both the linear ME effect (hence, ME multipoles) and second-order nonlinear magnetization. The present control method might be applicable to antiferromagnets belonging to these magnetic classes. In particular, the application to metallic antiferromagnets is of great interest. In metals, manipulation of ME multipoles by an electric field is severe challenge because of the screening of an applied electric field due to conduction electrons. Although an electric-current control of magnetic toroidal dipole order in metals has been recently demonstrated ${ }^{30-32}$, an analogous electric-current control of other two types of ME multipoles (i.e., magnetic monopole and quadrupole) cannot be anticipated because symmetries of these ME multipoles do not match with that of electric current ${ }^{32}$. Thus, the present electric-field-free method can be a useful way of controlling these ME multipoles in metals. It is also worthy to note that chirality can be externally induced by a mechanical twist in a nonchiral material, as observed in bismuth ${ }^{3}$. This can expand the applicability of the method to nonchiral antiferromagnets by simultaneously applying a mechanical twist and a magnetic field.

The present study reveals that PbTCPO possesses two different switchable (i.e., ferroic) orders: magnetic quadrupole and magnetic octupole orders. It can be viewed as a distinct type of multiferroic in which a coupling between these two orders gives rise to the observed unconventional cross-coupled phenomena. Moreover, the coupling originates from the presence of the crystal 
chirality. Further explorations of chirality-driven phenomena in $\mathrm{PbTCPO}$ and other chiral-lattice time-reversal-broken antiferromagnets are of great interest.

\section{METHODS}

\section{Sample preparation and characterization}

Single crystals of $\mathrm{Pb}(\mathrm{TiO}) \mathrm{Cu}_{4}\left(\mathrm{PO}_{4}\right)_{4}$ were grown by slow cooling of the melt ${ }^{20}$. The crystal chirality of grown crystals was distinguished by a sense of optical rotatory measured by means of polarized light microscopy, in which the incident light was parallel to the $Z$ axis and light wavelength was $500 \pm 10 \mathrm{~nm}$. We defined the crystal chirality of levo-rotatory crystals as $C$ - and that of dextro-rotatory crystals as $C+$. Monodomain crystals with $C+$ and $C$ - were used for electric polarization measurements described below. The orientation of the crystals was determined using the Laue X-ray photograph. The crystal structure displayed in Fig. 2a was drawn by using VESTA software ${ }^{33}$.

\section{Electric polarization measurements}

Electric polarization measurements on $\mathrm{C}+$ and $\mathrm{C}$-crystals were performed at the High Field Laboratory for Superconducting Materials in Tohoku University. Electric polarization along the $Y$ axis $\left(P_{Y}\right)$ as a function of temperature was obtained by integrating a displacement current measured with an electrometer (Keithley 6517). Before each measurement, the samples were cooled from $10 \mathrm{~K}\left(>T_{\mathrm{N}}\right)$ to $2 \mathrm{~K}\left(<T_{\mathrm{N}}\right)$ with or without a poling electric field $E_{\mathrm{p}}$, after which, if applied, $E_{\mathrm{p}}$ was switched off. Subsequently, the displacement current was measured on warming. To obtain ferroelectric hysteresis loops, i.e., the $E_{Y}$ dependence of $P_{Y}$, we applied a triangle wave voltage to the samples with a frequency of $5 \mathrm{mHz}$, which was generated by using a commercial voltage amplifier and a function generator. The electric polarization was obtained by integrating a displacement current induced by the voltage sweeping. In these measurements, a magnetic field was applied in the direction parallel to the $X Z$ plane, which makes an angle $-8^{\circ} \leq \Delta \theta \leq+8^{\circ}$ from the $X$ axis. The angle $\Delta \theta$ was controlled by rotating a sample stage about the $Y$ axis using a stepping motor.

\section{Theoretical calculations}

We employ the cluster mean-field theory of the chiral spin-1/2 model [Eq. (3)] for a microscopic understanding of the nonlinear magnetization in the system. In this treatment, the intercupola interactions are dealt with by the conventional mean-field approximation; namely $\mathbf{S}_{i} \cdot \mathbf{S}_{j}$ is decoupled as $\mathbf{S}_{i} \cdot \mathbf{S}_{j} \simeq\left\langle\mathbf{S}_{i}\right\rangle \cdot \mathbf{S}_{j}+\mathbf{S}_{i} \cdot\left\langle\mathbf{S}_{j}\right\rangle-\left\langle\mathbf{S}_{i}\right\rangle \cdot\left\langle\mathbf{S}_{j}\right\rangle$, while all the quantum fluctuations from the intracupola interactions and the Zeeman coupling term are fully taken into account by the exact diagonalization. In the calculation, we considered four cupolas in two unit cells; up and down cupolas in a single unit cell and those in a neighboring unit cell stacked along the $Z$ direction (see Supplementary Fig. 5). However, we always found solutions with the two unit cells identical to the single unit cell shown in Fig. 2a. This theoretical approach is suitable for the model analysis of cluster-based compounds, and has been used to qualitatively explain the magnetoelectric behavior in the series of compounds, $\mathrm{Ba}(\mathrm{TiO}) \mathrm{Cu}_{4}\left(\mathrm{PO}_{4}\right)_{4}{ }_{4}{ }^{25}, \mathrm{~Pb}(\mathrm{TiO})$ $\mathrm{Cu}_{4}\left(\mathrm{PO}_{4}\right)_{4}{ }^{21}$, and $\left.\mathrm{Sr}(\mathrm{TiO}) \mathrm{Cu}_{4}\left(\mathrm{PO}_{4}\right)_{4}\right)^{34}$.

\section{DATA AVAILABILITY}

The data that support the findings of this study are available from the corresponding author on request.

Received: 23 January 2021; Accepted: 11 May 2021; Published online: 31 May 2021

\section{REFERENCES}

1. Rikken, G. L. J. A. \& Raupach, E. Observation of magneto-chiral dichroism. Nature 390, 493-494 (1997).

2. Train, C. et al. Strong magneto-chiral dichroism in enantiopure chiral ferromagnets. Nat. Mater. 7, 729-734 (2008).

3. Rikken, G. L. J. A., Fölling, J. \& Wyder, P. Electrical magnetochiral anisotropy. Phys. Rev. Lett. 87, 236602 (2001).

4. Yokouchi, T. et al. Electrical magnetochiral effect induced by chiral spin fluctuations. Nat. Commun. 8, 866 (2017).
5. Seki, S. et al. Magnetochiral nonreciprocity of volume spin wave propagation in chiral-lattice ferromagnets. Phys. Rev. B 93, 235131 (2016).

6. Nomura, T. et al. Phonon magnetochiral effect. Phys. Rev. Lett. 122, 145901 (2019).

7. Hayami, S., Yatsushiro, M., Yanagi, Y. \& Kusunose, H. Classification of atomic-scale multipoles under crystallographic point groups and application to linear response tensors. Phys. Rev. B 98, 165110 (2018).

8. Suzuki, M.-T. et al. Multipole expansion for magnetic structures: a generation scheme for a symmetry-adapted orthonormal basis set in the crystallographic point group. Phys. Rev. B 99, 174407 (2019).

9. Astrov, D. N. The magnetoelectric effect in antiferromagnetics. Sov. Phys. JETP 11, 708-709 (1960).

10. Schmid, H. On ferrotoroidics and electrotoroidic, magnetotoroidic and piezotoroidic effects. Ferroelectrics 252, 41-50 (2001).

11. Fiebig, M. Revival of the magnetoelectric effect. J. Phys. D. Appl. Phys. 38, R123-R152 (2005)

12. Spaldin, N. A., Fiebig, M. \& Mostovoy, M. The toroidal moment in condensedmatter physics and its relation to the magnetoelectric effect. J. Phys. Condens. Matter 20, 434203 (2008).

13. Kimura, $\mathrm{K}$. et al. Magnetodielectric detection of magnetic quadrupole order in $\mathrm{Ba}$ (TiO) $\mathrm{Cu}_{4}\left(\mathrm{PO}_{4}\right)_{4}$ with $\mathrm{Cu}_{4} \mathrm{O}_{12}$ square cupolas. Nat. Commun. 7, 13039 (2016).

14. Kimura, K., Katsuyoshi, T., Sawada, Y., Kimura, S. \& Kimura, T. Imaging switchable magnetoelectric quadrupole domains via nonreciprocal linear dichroism. Commun. Mater. 1, 39 (2020).

15. Watanabe, H. \& Yanase, Y. Magnetic hexadecapole order and magnetopiezoelectric metal state in $\mathrm{Ba}_{1-x} \mathrm{~K}_{x} \mathrm{Mn}_{2} \mathrm{As}_{2}$. Phys. Rev. B 96, 064432 (2017).

16. Lovesey, S. W., Khalyavin, D. D. \& Staub, U. Ferro-type order of magneto-electric quadrupoles as an order-parameter for the pseudo-gap phase of a cuprate superconductor. J. Phys. Condens. Matter 27, 292201 (2015).

17. Fechner, M., Fierz, M. J. A., Thöle, F., Staub, U. \& Spaldin, N. A. Quasistatic magnetoelectric multipoles as order parameter for pseudogap phase in cuprate superconductors. Phys. Rev. B 93, 174419 (2016).

18. Hlinka, J. Eight types of symmetrically distinct vectorlike physical quantities. Phys. Rev. Lett. 113, 165502 (2014).

19. Cheong, S.-W., Lim, S., Du, K. \& Huang, F.-T. Permutable SOS (symmetry operational similarity). Preprint at bioRxiv https://arxiv.org/abs/2101.03123 (2021).

20. Kimura, $\mathrm{K}$. et al. A-cation control of magnetoelectric quadrupole order in $A(\mathrm{TiO}) \mathrm{Cu}_{4}\left(\mathrm{PO}_{4}\right)_{4}(A=\mathrm{Ba}, \mathrm{Sr}$, and $\mathrm{Pb})$. Phys. Rev. B 97, 134418 (2018).

21. Kimura, K. et al. Magnetic structural unit with convex geometry: a building block hosting an exchange-striction-driven magnetoelectric coupling. Phys. Rev. Mater. 2, 104415 (2018).

22. Aroyo, M. I. et al. Bilbao crystallographic server: I. Databases and crystallographic computing programs. Z. Kristallogr. 221, 15-27 (2006).

23. Aroyo, M. I., Kirov, A., Capillas, C., Perez-Mato, J. M. \& Wondratschek, H. Bilbao crystallographic server. II. Representations of crystallographic point groups and space groups. Acta Crystallogr. A62, 115-128 (2006).

24. Tokunaga, Y., Taguchi, Y., Arima, T. \& Tokura, Y. Magnetic biasing of a ferroelectric hysteresis loop in a multiferroic orthoferrite. Phys. Rev. Lett. 112, 037203 (2014).

25. Kato, Y. et al. Magnetoelectric behavior from $S=1 / 2$ asymmetric square cupolas. Phys. Rev. Lett. 118, 107601 (2017).

26. Harris, A. B. Landau analysis of the symmetry of the magnetic structure and magnetoelectric interaction in multiferroics. Phys. Rev. B 76, 054447 (2007).

27. Harris, A. B. Identifying Landau order parameters and their transformation properties for complex multiferroics: the case of $\mathrm{Mn}_{2} \mathrm{GeO}_{4}$. Phys. Rev. B 96, 054422 (2017).

28. Hayashida, T., Kimura, K., Urushihara, D., Asaka, T. \& Kimura, T. Observation of ferrochiral transiiton induced by an antiferroaxial ordering of antipolar structural units in $\mathrm{Ba}(\mathrm{TiO}) \mathrm{Cu}_{4}\left(\mathrm{PO}_{4}\right)_{4}$. J. Am. Chem. Soc. 143, 3638-3646 (2021).

29. Generators/General Positions 3D Crystallographic Point Groups. Bilbao Crystallographic Server https://www.cryst.ehu.es/cryst/get_point_genpos.html.

30. Godinho, J. et al. Electrically induced and detected Néel vector reversal in a collinear antiferromagnet. Nat. Commun. 9, 4686 (2018).

31. Watanabe, H. \& Yanase, Y. Symmetry analysis of current-induced switching of antiferromagnets. Phys. Rev. B 98, 220412 (2018).

32. Thöle, F., Keliri, A. \& Spaldin, N. A. Concepts from the linear magnetoelectric effect that might be useful for antiferromagnetic spintronics. J. Appl. Phys. 127, 213905 (2020).

33. Momma, K. \& Izumi, F. VESTA 3 for three-dimensional visualization of crystal, volumetric and morphology data. J. Appl. Crystallogr. 44, 1272-1276 (2011).

34. Kato, Y. et al. Magnetoelectric behavior from cluster multipoles in square cupolas: study of $\mathrm{Sr}(\mathrm{TiO}) \mathrm{Cu}_{4}\left(\mathrm{PO}_{4}\right)_{4}$ in comparison with $\mathrm{Ba}$ and $\mathrm{Pb}$ isostructurals. Phys. Rev. B 99, 024415 (2019).

\section{ACKNOWLEDGEMENTS}

We wish to thank T. Katsuyoshi for his help in the electric polarization measurement. We also thank S. Hayami for fruitful discussion on the symmetry argument of nonlinear spin susceptibility. This work was partially supported by JSPS KAKENHI 
Grant Numbers JP17H02917, JP17H01143, JP18K03447, JP19H05823, JP19H05825, and JP19H01847, by the MEXT Leading Initiative for Excellent Young Researchers (LEADER) and by JST CREST (JP-MJCR18T2). This work was partly performed at the High Field Laboratory for Superconducting Materials, Institute for Materials Research Tohoku University (Project No $18 \mathrm{H} 0014$ and 19H0009). Numerical calculations were performed using the facilities of the Supercomputer Center, the Institute for Solid State Physics, the University of Tokyo.

\section{AUTHOR CONTRIBUTIONS}

K.K. and T.K. conceived the project. K.K. and S.K. coordinated experiments. K.K. grew single crystals and measured electric polarization. Y.K. and Y.M. performed theoretica calculations and cluster multipole decomposition analysis. K.K., Y.K., Y.M., and T.K. wrote the paper.

\section{COMPETING INTERESTS}

The authors declare no competing interests.

\section{ADDITIONAL INFORMATION}

Supplementary information The online version contains supplementary material available at https://doi.org/10.1038/s41535-021-00355-0.
Correspondence and requests for materials should be addressed to K.K.

Reprints and permission information is available at http://www.nature.com/ reprints

Publisher's note Springer Nature remains neutral with regard to jurisdictional claims in published maps and institutional affiliations.

Open Access This article is licensed under a Creative Commons Attribution 4.0 International License, which permits use, sharing, adaptation, distribution and reproduction in any medium or format, as long as you give appropriate credit to the original author(s) and the source, provide a link to the Creative Commons license, and indicate if changes were made. The images or other third party material in this article are included in the article's Creative Commons license, unless indicated otherwise in a credit line to the material. If material is not included in the article's Creative Commons license and your intended use is not permitted by statutory regulation or exceeds the permitted use, you will need to obtain permission directly from the copyright holder. To view a copy of this license, visit http://creativecommons. org/licenses/by/4.0/.

(c) The Author(s) 2021 